\title{
Spectrum Fitting Code for LAMOST ExtraGAlactic Surveys (LEGAS)
}

\author{
Xu Kong ${ }^{1,2} \&$ Shanshan $\mathbf{S u}^{2}$ \\ ${ }^{1}$ Key Laboratory for Research in Galaxies and Cosmology, University of Science and \\ Technology of China (USTC), CAS, China \\ email: xkong@ustc.edu.cn \\ ${ }^{2}$ Center for Astrophysics, USTC, Hefei, Anhui, 230026, China
}

\begin{abstract}
The study of stellar populations in galaxies is entering a new era with the availability of large and high-quality data bases of both observed galactic spectra and state-of-the-art evolutionary synthesis models. The Large Sky Area Multi-Object Fiber Spectroscopic Telescope (LAMOST) has a $4 \mathrm{~m}$ primary, and a 5 degree field of view, accommodate as many as 4000 optical fibers. It can obtain the spectra of objects as faint as down to $B=20.5$ mag with an exposure of 1.5 hour, promising a very high spectrum acquiring rate of ten-thousands of spectra per night. In this talk, we introduce the progress of the LAMOST project, its surveys and show the spectral synthesis code that we are developing for the LAMOST ExtraGAlactic Surveys (LEGAS).
\end{abstract}

Keywords. methods: data analysis, galaxies: fundamental parameters, galaxies: stellar content

\section{What is the LAMOST}

The Large Sky Area Multi-Object Fiber Spectroscopic Telescope (LAMOST) is a telescope being built in China. It is a reflecting Schmidt telescope with a 4-meter aperture and a 5 degree field of view. It has as many as 4000 optical fibers which can collect up to 4000 spectra during one exposure. The characteristics of LAMOST are: (1) it is a meridian reflecting Schmidt telescope with an active correcting plate; (2) with both wide field of view (5 degrees) and large aperture (effective in 4 meters); (3) there are 4000 optical fibers on the focal surface for obtaining 4000 spectra; (4) 16 spectrographs with resolution $R=1000 / 2000$ for low with wave band 3700-5900 $\AA$ in blue and 5700-9000 $\AA$ in red.

With its 1.5 hours average exposure time, and $5 \AA$ spectral resolution, the $B=20.5$ magnitude objects could be observed in LAMOST spectroscopic survey. Since 4000 spectra of objects could be obtained during one observation, LAMOST will have the highest spectrum acquiring rate for the wide field and large sample astronomy. It will make substantial contribution to the study of structure and evolution of our Galaxy, galaxies formation and evolution and the large-scale structure of the universe (Cui et al. 2009).

LAMOST has been completed the whole system and engineering test in the end of 2008; start the technical commissioning with complete system in 2009; the scientific commissioning with the pilot surveys in 2010, and the regular spectroscopic survey in the beginning of 2011 .

\section{What is the LAMOST ExtraGAlactic Surveys}

While many important achievements in large scale structure and galaxy formation have been made by recent galaxy redshift surveys such as the 2dFGRS and SDSS, many key 


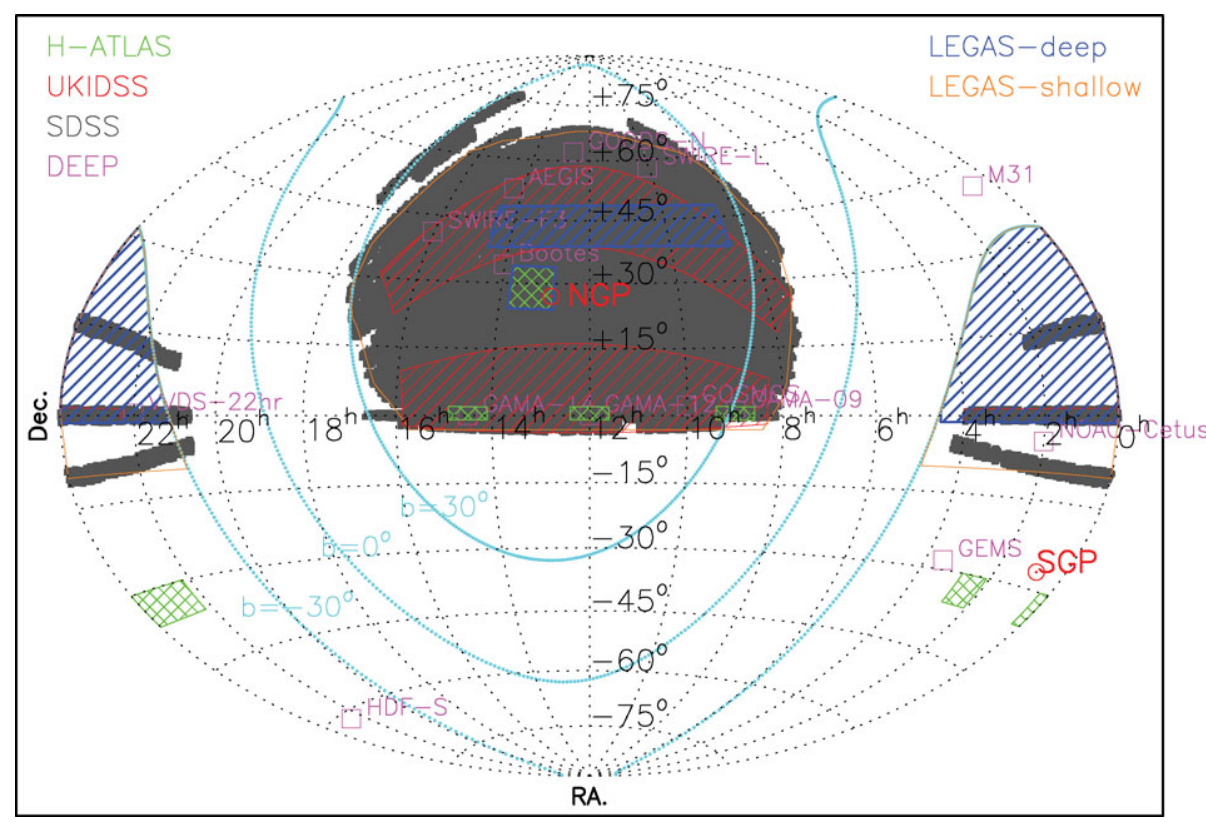

Figure 1. The sky map of the LAMOST ExtraGAlactic Surveys. The boundaries of the LEGAS areas and other surveys are illustrated as different color lines.

scientific questions remain unanswered. Compared with the telescope used for the SDSS and 2dFGRS, the LAMOST telescope has a large aperture, a large field of view, and more importantly is quipped with 4000 fibers, will probe a larger volume and observe fainter galaxies and quasars than SDSS and 2dFGRS. With the LAMOST, we will be able to complete, in a reasonable time frame (about 5 years), surveys of galaxies and quasars (LAMOST ExtraGAlactic Surveys, LEGAS) which exceed existing surveys by a factor of $5 \sim 10$ in the number of objects and in the volume probed, thus help solving many outstanding problems in cosmology and galaxy.

LEGAS includes the following three spectroscopic surveys. 1) LEGAS-deep: spectroscopic survey of 3.5 million galaxies to a magnitude $r=19.5$ in the areas of 3500 square degrees. The spectra will be taken with the exposure time of 1.5 hours during dark nights; 2) LEGAS-shallow: spectroscopic survey of 2.4 million galaxies to a magnitude $r=18.7$ in the area of 8100 square degrees other than that covered by the LEGAS-deep. The spectra will be taken with the exposure time of 30 minutes; 3) LEGAS-QSOs: Spectroscopic Survey of 0.6 million quasars to a magnitude $i=20.5$, with the exposure time set to be the same as those of LEGAS-deep and LEGAS-shallow in the same sky area. The boundaries of the LEGAS sky areas are illustrated in Figure 1.

For these three surveys, LEGAS-shallow is concentrated on the questions about the physical properties of dark energy, dark matter and large scale structure formation; LEGAS-QSOs is concentrated on the questions about the growth of super massive black holes and the co-evolution with their host galaxies. LEGAS-deep, with the advantage of wide area coverage $\left(3500 \mathrm{deg}^{2}\right)$ and deep limited magnitude $(r=19.5)$, allow us to investigate a well-defined galaxy sample at redshifts intermediate between $z>0.6$ distant galaxies and the local galaxies $(z<0.2)$. This redshift range $(0.2<z<0.6)$ is very important to understand evolution of galaxies in the field and group environment. 


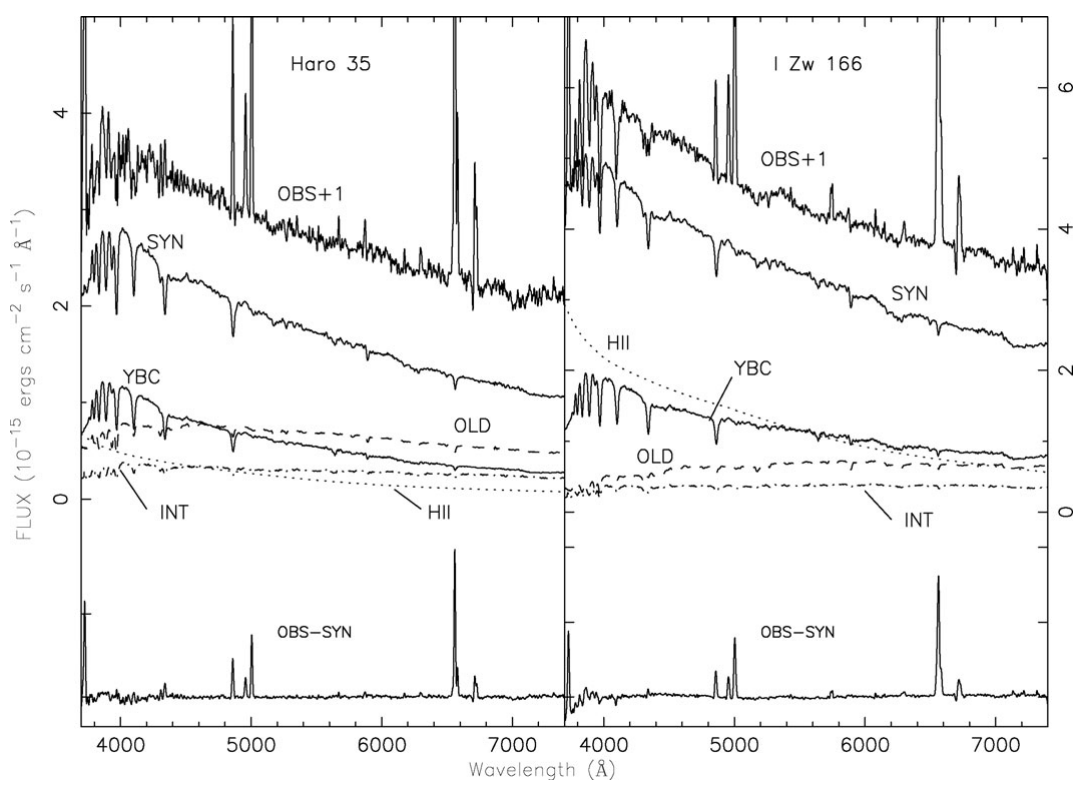

Figure 2. Examples of spectral fitting for two galaxies in Kong et al. (2003). OBS is the observed spectrum of galaxy, SYN is the synthesis stellar spectrum from the best matched template, OBS-SYN is the difference between them. Components of the synthesis grouped into old (OLD), intermediate age (INT), young (YBC), and HII (HII), shown to scale according to their flux fraction contributions at $5870 \AA$.

\section{Spectrum Fitting Code for Galaxies in LEGAS}

The global properties of galaxies are determined by the nature and evolution of their stellar components. Stellar populations keep a record of most of the evolution of galaxies, therefore, spectral analysis of stellar populations of galaxies can be used to determine the properties (such as dust content, stellar velocity dispersion and metallicity) and constrain the history of the star formation and enrichment since their formation. In additional, emission lines in the spectrum of galaxy are important tools for studying star formation and chemical evolution in galaxies, but they are often heavily contaminated with underlying stellar absorption. Proper decomposition of emission lines and starlight components is required for a physical interpretation of a galaxy spectrum. Because only few galaxies can be resolved into individual stars, population synthesis method is often used to analyze stellar population of galaxy and to subtract underlying stellar absorption from nebular emission lines. In our Center (Center for astrophysics, USTC), since 1999, we have used and developed different population synthesis methods, which are used to analyze the spectral properties for galaxies (Kong \& Cheng 1999; Kong et al. 2000, Kong et al. 2003, Li et al. 2005, Lu et al. 2005). In Figure 2, as an example, we show the spectral fitting results for two galaxies in Kong et al. (2003).

LAMOST is a unique astronomical instrument in combining a large aperture with a wide field of view. With 4000 fibers, LAMOST will observe several ten-thousands of spectra per night. To determine the characteristics of stellar population in galaxies and also can subtract the underlying stellar absorption from emission lines, we are developing a new version spectrum fitting code for galaxy spectra, which will be observed by the LEGAS project. In this code, three special attentions are given to improve efficiency of the code, to compare difference from different stellar libraries and different fitting algorithms. 
Firstly, to reduce the input spectral templates, and then can improve the fitting efficiency, independent component analysis method and principal component analysis method were used recently by $\mathrm{Li}$ et al. (2005) and Lu et al. (2006), respectively. These methods can sufficiently compress the synthetic galaxy spectral library to less then 10 principal components or independent components, which be used as input templates for spectral fitting. But the classical methods often use several hundred spectra, from evolutionary population synthesis code, as input templates. The former two methods can greatly reduce the computational time of the fit and is ideal for fitting large data sets like our LEGAS. But before we apply them, we have to check them very carefully, to compare the results from these two methods, and also from those classical methods.

Secondly, spectrum fitting code will use templates from evolutionary population synthesis code, which are affected by uncertainties in the stellar evolutionary tracks and in the stellar library used to construct them. Despite permanent progress, some aspects of stellar evolution remain difficult to model (e.g. the asymptotic giant branch, the red supergiant phase; effects of a binary companion). The input library of stellar spectra can be either empirical or theoretical. The shortcomings of theoretical spectra are the uncertainties of atomic and molecular data; the disadvantages of empirical spectra are the limited parameter set $(\mathrm{T}, \mathrm{g}, \mathrm{Z})$ with a finite SNR. There exists several such codes, and in our work we are using Pegase-HR, Galaxev (BC03 and CB07) and Vazdekis/Miles, to investigate the effects from evolutionary population synthesis models.

Finally, spectrum fitting method consists of minimizing the $\chi^{2}$ between an observed spectrum and a model. The results are affected by spectrophotometric and wavelength calibration error, and model error, along with effects of the age-metallicity-extinction degeneracy. Therefore, the fitting algorithms are very important to the final results. Some methods, such as MOPED (Heavens et al. 2000), Platefit/MPA-JHU (Brinchmann et al. 2004), STARLIGHT (Cid Fernandes et al. 2005), STEC(K)MAP (Ocvirk et al. 2006), VESPA (Tojeiro et al. 2007), and ULySS (Koleva et al. 2009), have been developed in these years, we will check the results from some of these methods, and develop our spectrum fitting code for LEGAS.

\section{Acknowledgements}

The work is supported by the National Natural Science Foundation of China (NSFC, Nos. 10633020, and 10873012), the Knowledge Innovation Program of the Chinese Academy of Sciences (No. KJCX2-YW-T05), and National Basic Research Program of China (973 Program; No. 2007CB815404).

\section{References}

Brinchmann, J., Charlot, S., White, S. D. M., et al. 2004, MNRAS, 351, 1151

Bruzual, G. \& Charlot, S. 2003, MNRAS, 344, 1000

Cid Fernandes, R., Mateus, A., Sodré, L., et al. 2005, MNRAS, 358, 363

Cui, X. 2009, Bulletin of the American Astronomical Society, 41, 473

Heavens, A. F., Jimenez, R., \& Lahav, O. 2000, MNRAS, 317, 965

Koleva, M., Prugniel, P., Bouchard, A., \& Wu, Y. 2009, A\&A, 501, 1269

Kong, X. \& Cheng, F. Z. 1999, A\& A, 351, 477

Kong, X., Charlot, S., Weiss, A., \& Cheng, F. Z. 2003, A\&A A, 403, 877

Li, C., Wang, T.-G., Zhou, H.-Y., et al. 2005, AJ, 129, 669

Lu, H., Zhou, H., Wang, J., et al. 2006, AJ, 131, 790

Ocvirk, P., Pichon, C., Lançon, A., et al. 2006, MNRAS, 365, 74

Tojeiro, R., Heavens, A. F., Jimenez, R., et al. 2007, MNRAS, 381, 1252 